\title{
Letrozole regulates actin cytoskeleton polymerization dynamics in a SRC-1 dependent manner in the hippocampus of mice
}

\author{
Yangang Zhao ${ }^{1 *}$, Yanlan $\mathrm{Yu}^{2 *}$, Yuanyuan Zhang ${ }^{1}, \mathrm{Li} \mathrm{He}^{3}$, Linli Qiu ${ }^{3,4}$, Jikai Zhao ${ }^{1}$, Mengying \\ Liu $^{1}$, Jiqiang Zhang ${ }^{1 * *}$
}

1. Department of Neurobiology, Chongqing Key Laboratory of Neurobiology, Third Military Medical University, Chongqing 400038, China

2. Student Brigade, Third Military Medical University, Chongqing 400038, China

3. School of Nursing, Third Military Medical University, Chongqing 400038, China

4. Department of Nursing, Sichuan Nursing Vocational College, Chengdu 610100, China

* Equal contribution.

** Corresponding author. Dr. Zhang J: Department of Neurobiology, Third Military Medical University, Chongqing 400038, China. Tel.: +86 23 68752223, Email: zhangjqtmmu @ yahoo.com.

\begin{abstract}
In the hippocampus, local estrogens (E2) derived from testosterone that is catalyzed by aromatase play important roles in the regulation of hippocampal neural plasticity, but the underlying mechanisms remain unclear. The actin cytoskeleton contributes greatly to hippocampal synaptic plasticity; however, whether it is regulated by local E2 and the related mechanisms remain to be elucidated. In this study, we first examined the postnatal developmental profiles of hippocampal aromatase and specific proteins responsible for actin cytoskeleton dynamics. Then we used aromatase inhibitor letrozole (LET) to block local E2 synthesis and examined the changes of these proteins and steroid receptor coactivator-1 (SRC-1), the predominant coactivator for steroid nuclear receptors. Finally, SRC-1 specific RNA interference was used to examine the effects of SRC-1 on the expression of these actin remodeling proteins. The results showed a Vtype profile for aromatase and increased profiles for actin cytoskeleton proteins in both male and female hippocampus without obvious sex differences. LET treatment dramatically decreased the F-actin/G-actin ratio, the expression of Rictor, phospho-AKT (ser473), Profilin-1, phosphoCofilin (Ser3), and SRC-1 in a dose-dependent manner. In vitro studies demonstrated that LET induced downregulation of these proteins could be reversed by E2, and E2 induced increase of these proteins were significantly suppressed by SRC-1 shRNA interference. These results for the first time clearly demonstrated that local E2 inhibition could induce aberrant actin polymerization; they also showed an important role of SRC-1 in the mediation of local E2 action on hippocampal synaptic plasticity by regulation of actin cytoskeleton dynamics.
\end{abstract}

Keyword: Hippocampus; Estrogens; Aromatase; Letrozole; Steroid receptor coactivator-1; Actin Cytoskeleton

\section{Introduction}

Estrogens, especially $17 \beta$-estradiol (E2), have been shown to play important roles in the regulation of the structure and function of the hippocampus [1]. In addition to estrogen coming from peripheral circulation, such as the ovaries, hippocampal E2 (local E2) can also be de novo 
synthesized from cholesterol that is catalyzed by aromatase (AROM), the final limiting enzyme for estrogen synthesis [2-4]. Accumulated studies have reported this local E2 plays a pivotal role in the regulation of hippocampal synaptic plasticity as well as spatial learning and memory. For example, it has been shown that the density of spine synapses in hippocampal slices depends on local E2 formation [5], and hippocampal synapse formation (synaptogenesis) needs the conversion of cholesterol to E2 [6]. This local E2 also plays an important role in the induction phase of high-frequency stimulation (HFS)-dependent long-term potentiation (LTP), as shown by letrozole (LET), a reversible nonsteroidal AROM inhibitor [7]. These results were further demonstrated by Vierk et al., who showed that LET treatment induced strong and significant impairment of the LTP and loss of hippocampal spine synapses [8]. Another study by Bayer et al. showed that LET treatment specifically impaired hippocampus-dependent memory in both rodents and post-menopausal women [9].

Dendritic spines are specialized structures of the postsynaptic membrane receiving glutamatergic synaptic inputs, which is deeply related to synaptic plasticity and learning [10] and the actin cytoskeleton is the structural element underlying its proper development and morphology [11]. Actin filaments rapidly shift between polymerization (F-actin) and depolymerization (G-actin) states, in which F-actin is a polymer built up from G-actin [12]. Several proteins, such as Cofilin (responsible for actin depolymerization), Profilin-1 (responsible for actin polymerization) [13], and Rictor (the rapamycin-insensitive companion of mTOR, functions to regulate the balance of Profilin/Cofilin components) [14] have been shown to be profoundly involved in the regulation of actin cytoskeleton dynamics. Abundant studies have reported that, in the hippocampus, peripheral E2 treatment increases dendritic spine density [8] and LET treatment induces significant loss in the density of spine synapses and in the number of presynaptic buttons as well as selected synaptic proteins $[5,15,16]$. However, whether and how this regulation involves actin remodeling proteins is not clear.

Rare studies have reported the developmental profile of AROM in the hippocampus [17, 18]. The profiles of key actin cytoskeleton regulatory proteins in the hippocampus after birth and whether they are regulated by local E2 are also unclear. To address these questions, in this study, we first examined the postnatal changes of hippocampal AROM as well as the above mentioned actin remodeling proteins, and then we examined the effects of local E2 inhibition on the expression of these actin cytoskeleton remodeling proteins using LET and/or E2 treatment. Finally, since steroid receptor coactivator-1 (SRC-1; which is needed for the transcriptional activity of steroid nuclear receptors [19]) has been shown to mediate LET regulation on hippocampal synaptic protein PSD-95 [15], we then explored the roles of SRC-1 in the LET/E2 regulation on these actin remodeling proteins after SRC-1 knockdown using specific shRNA against SRC-1.

\section{Materials and Method}

\subsection{Animals and LET Administration}

Different postnatal (P0-P56) male and female and adult (12 weeks old, $20 \pm 2 \mathrm{~g}$ ) female SPF grade C57BL/6 mice were obtained from the Experimental Animal Center of the Third Military Medical University. LET (L6545, Sigma-Aldrich Shanghai Trading Co Ltd, Shanghai, China) was dissolved in DMSO (D5879, Sigma-Aldrich Shanghai Trading Co Ltd, Shanghai, China) and diluted with sterile saline solution before injection. Adult female SPF grade C57BL/6 mice were 
randomly divided into four groups. The LET groups were intraperitoneally injected with LET in doses of $40 \mu \mathrm{g} / \mathrm{kg}, 80 \mu \mathrm{g} / \mathrm{kg}$ and $160 \mu \mathrm{g} / \mathrm{kg}$ body weight (100 $\mu \mathrm{l} / \mathrm{each}$ mouse), respectively. The control animals were injected intraperitoneally with vehicle solution (2\% DMSO + 98\% sterile saline solution; $100 \mu \mathrm{l} /$ each mouse). The injection was conducted every morning and lasted for 1 week. At day 7, all of the animals were killed for further experiments. All of the animal-related procedures were conducted in strict compliance with the Approved Institutional Animal Care and Use Protocols.

\subsection{SRC-1 RNA Interference Lentivirus Preparation}

For lentiviral gene transfection, lentiviruses that expressed enhanced GFP and SRC-1 under the control of the pLentis-EF-GFP-miR-PGK-PURO parent vector were used. To make the SRC-1 shRNA compatible with multiple viral vectors, a ligase was used to insert the shRNA into the PSTI site of the vectors. Three SRC-1 shRNAs were designed and tested; the sequences of these shRNAs were as follows:

shRNA-1:

sense:

TGCTGTTGACAGTGAGCGCGCCCATCCTATAGATTCATATTAGTGAAGCC $\mathrm{AC}$;

antisense: AGATGTAATATGAATCTATAGGATGGGCTTGCCTACTGCCTCGGA.

shRNA-2:

sense:

TGCTGTTGACAGTGAGCGACCTCTTTAAACCTCAATAATTTAGTGAAGCC

A; antisense: CAGATGTAAATTATTGAGGTTTAAAGAGGGTGCCTACTGCCTCGGA. shRNA-3:

sense:

TGCTGTTGACAGTGAGCGCGCAGATTGCCTGAACTAGAATTAGTGAAGCC A;

antisense: CAGATGTAATTCTAGTTCAGGCAATCTGCTTGCCTACTGCCTCGGA.

The human elongation factor-1 alpha (EF-1 $\alpha)$ promoter and the SRC-1 shRNA were cloned into the Xhol/EcoRI site of the vectors. Because the three SRC-1 shRNAs produced similar interference efficacy in 293T cells (data not shown), we selected shRNA-1 (referred to as shSRC1) for use in subsequent experiments.

For viral particle production, HEK 293T/17 cells were grown in DMEM/10\% fetal bovine serum (FBS) and plated in 10-cm dishes at a density of $3 \times 10^{6}$ cells/dish. The day after plating, the cells were transfected with SRC-1 shRNA plasmids using calcium phosphate as follows: $1.5 \mu \mathrm{g}$ pMD2G, $4.5 \mu \mathrm{g}$ psPAX2, and $6 \mu \mathrm{g}$ multiple viral vectors. The conditioned medium was collected $48 \mathrm{~h}$ after transfection and immediately frozen for the preparation of SRC-1-containing particles. For the second harvest of viral particles, which took place on the next day, DMEM was replaced with fresh medium. The supernatant was clarified by filtering it through a $0.45-\mu \mathrm{m}$ filter and was ultracentrifuged at $4^{\circ} \mathrm{C}$ for 120 minutes at $50,000 \mathrm{~g}$. The pellet was resuspended in $100 \mu \mathrm{l}$ PBS and left standing at $4^{\circ} \mathrm{C}$ for 30 minutes. Viral titer, in transducing units (TU)/ml, was determined using a human immunodeficiency virus (HIV) p24 ELISA kit (ZeptoMetrix Corporation, Buffalo, NY) and a Lentivirus Quantitation kit (Cell Biolabs) in HEK-293T cells [20]; and the final titer was $1.5 \times 10^{9} \mathrm{TU} / \mathrm{ml}$. 


\subsection{Cell Culture, LET and $\mathrm{E}_{\mathbf{2}}$ Treatment and siSRC-1 Lentivirus Transfection}

Cells of the embryonic mouse hippocampal cell line mHippoE-14 (CELLutions Biosystems Inc, Canada) [21] were cultured in DMEM with 10\% fetal bovine serum (Gibco, 10099-141), 25 $\mathrm{mM}$ glucose and $1 \%$ penicillin/streptomycin and maintained at $37^{\circ} \mathrm{C}$ with $5 \% \mathrm{CO}_{2}$ in a humidified chamber.

LET and 17ß-estradiol (E2; ab120657, Abcam Shanghai Trading Co Ltd, Shanghai, China) was dissolved in DMSO. The final concentration of LET was $10^{-6}, 10^{-8}$ and $10^{-10} \mathrm{~mol} / \mathrm{L}(\mathrm{M})$ and the final concentration of E2 was $10^{-6} \mathrm{~mol} / \mathrm{L}(\mathrm{M})$. For LET and/or E2 treatment, $2 \mu 1$ of LET or E2 in DMSO (control) was added to one well of the six-well plates and incubated for 2 days. For siSRC-1 transfection, cells plated in six-well plates were transduced for $48 \mathrm{~h}$ with $5 \mu \mathrm{l}$ of lentivirus; then the medium was replaced with fresh DMEM containing an appropriate concentration of puromycin and the screened cells were used for subsequent experiments. The untreated normal mHippoE-14 cells and the empty lentiviral vectors in treated cells were used as controls. All of these experiments were repeated at least three times.

\subsection{Western Blot Analysis}

Western blot analysis was carried out according to our previous reports $[15,22,23]$. The hippocampus of the mice or from mHippoE-14 cells were lysed, membranes were blocked with 5\% milk-TBST and then incubated with the individual diluted primary antibodies at $4{ }^{\circ} \mathrm{C}$ overnight. After several washes, the membranes were incubated with HRP-conjugated goat antirabbit secondary antibodies (1:2,000, ZB-2301, Zhongshan Biotech) or goat anti-mouse secondary antibodies (1:2,000, ZB-2305, Zhongshan Biotech). The blots were finally visualized with chemiluminescent HRP substrate (WBKLS0100, MerkMillipore) by Western Lightning-ECL (Bio-Rad, Hercules, USA). For phospho-Akt (Ser473) /AKT or phospho-Cofilin (Ser3)/Cofilin measurement, the two phospho-proteins were examined first with the protocol as mentioned above, then the membranes were washed with a specific stripping buffer (P0023A, Beyotime Biotech), blocked with 5\% fresh-prepared milk, incubated with the diluted individual primary antibodies against AKT or Cofilin, and then, the process continued to the next step. The optical density for each band was measured using Quantity One software (Bio-Rad) and normalized to that of $\beta$-actin or corresponding total proteins. A blank control (without the primary antibody) was used to determine the specificity of the primary antibodies and a pre-stained protein marker was employed to localize the specific band of the primary antibodies.

The antibodies were as follows: mouse monoclonal anti-aromatase (1:500; SM2222PS, Acris antibodies, Inc., US); rabbit polyclonal anti-SRC-1 (1:600; sc-8995, Santa Cruz), rabbit mAbRictor (1:500; 2140, Cell Signaling), rabbit mAb-phospho-Akt (Ser473) (1:2,000; 9271, Cell Signaling), rabbit mAb-Akt (1:1,000; 9272, Cell Signaling), rabbit monoclonal actin antibody (1:2,000; GTX61452, GeneTex), rabbit mAb-phospho-Cofilin (Ser3) (1:300; 3311, Cell Signaling), rabbit mAb-Cofilin (1:300; 3312, Cell Signaling), rabbit polyclonal anti-Profilin-1 (1:4,000; GTX63456, GeneTex), and mouse mAb- $\beta$-actin (1:1,000, AA128, Beyotime Biotech).

\subsection{F-actin/G-actin Ratio Measurement}

The F-actin to G-actin ratio was determined by Western blotting, as previously described [14] Briefly, the hippocampus was isolated and homogenized in cold lyses buffer $\left(10 \mathrm{mM} \mathrm{K} \mathrm{HPO}_{4}\right.$, 
$100 \mathrm{mM} \mathrm{NaF}, 50 \mathrm{mM} \mathrm{KCl}, 2 \mathrm{mM} \mathrm{MgCl} 2,1 \mathrm{mM}$ EGTA, $0.2 \mathrm{mM}$ dithiothreitol, $0.5 \%$ Triton X$100,1 \mathrm{M}$ sucrose, $\mathrm{pH} 7.0$ ) and then centrifuged at 15,000 $\mathrm{g}$ for $30 \mathrm{~min}$. G-actin (soluble actin) was measured in the supernatant with a rabbit monoclonal actin antibody. To measure F-actin, the pellets were resuspended in an equal volume of lyses buffer $(1.5 \mathrm{mM}$ guanidine hydrochloride, 1 $\mathrm{mM}$ sodium acetate, $1 \mathrm{mM} \mathrm{CaCl}, 1 \mathrm{mM} \mathrm{ATP}, 20 \mathrm{mM}$ Tris-HCl, $\mathrm{pH} 7.5)$ and incubated on ice for $1 \mathrm{~h}$ to depolymerize F-actin with gentle shaking every $15 \mathrm{~min}$. The samples were centrifuged at $15,000 \mathrm{~g}$ for $30 \mathrm{~min}$, and this supernatant was used to measure actin (as a reflection of insoluble Factin) with the same rabbit monoclonal actin antibody. Samples from the supernatant (G-actin) and pellet (F-actin) fractions were proportionally loaded and analyzed with Western blot as mentioned above.

\subsection{Statistical Analysis}

All of the results are shown as the mean \pm S.E. A one-way ANOVA followed by LSD-test was conducted using SPSS software (version 13.0, IBM; Chicago, USA), and $p<0.05$ was considered statistically significant.

\section{Results}

\subsection{Postnatal Profile of AROM and Actin Regulatory Proteins in the Hippocampus}

We first analyzed levels of AROM protein in the postnatal hippocampus of male and female mice. Western blot analysis showed that, in the male hippocampus, high levels of AROM were detected at $\mathrm{P} 0$ and $\mathrm{P} 7$, and there were no significant differences between these two checkpoints ( $p>0.05)$. After P7, it decreased dramatically at P14 and P28 when compared with that of P7 $(\mathrm{p}<0.01)$. At P56, it increased significantly when compared to P28 $(\mathrm{p}<0.01)$, but showed no statistical significance when compared with P0 or P7 (p>0.05). A similar V-type postnatal profile was detected in the female hippocampus, higher levels of AROM were detected at P0 and P7, then it decreased from P14 to P28, there was no significant difference detected between P0 and P7, or between P14 and P28 (p>0.05). However, at P56 it increased to the highest level when compared with any other checkpoint $(\mathrm{p}<0.01)$, which was different from that in the males (Fig. 1A).

We then analyzed the expression of Rictor in the hippocampus. The results showed that both males and females shared an identical postnatal profile. In detail, the lowest levels were detected at P0, and then it increased with development. Significantly higher levels of Rictor were detected at P7 ( $<<0.05$ when compared with P0) and then it reached the highest levels at P14. No significant differences were detected among P14, P28 and P56 ( $>0.05$ ). These results were shown in Fig. 1B.

As to hippocampal p-Cofilin and Cofilin, both males and females showed similar postnatal profiles. Because Cofilin was almost unchanged but $\mathrm{p}$-Cofilin changes significantly after birth, the ratio of $\mathrm{p}$-Cofilin/Cofilin was the lowest at $\mathrm{P} 0$, and then, this ratio increased slightly at $\mathrm{P} 7$ and reached the highest levels at $\mathrm{P} 28$ ( $\mathrm{p}<0.01$ when compared with $\mathrm{P} 0, \mathrm{P} 7$ and P14). There was no statistical significance among P0, P7 and P14 (p>0.05). However, at P56 it was slightly decreased when compared with that of $\mathrm{P} 28$ ( $\mathrm{p}<0.05$ in the males and $\mathrm{p}<0.01$ in the females). These results were shown in Fig. 1C.

As to Profilin-1, both males and females shared similar ontogenetic profiles. Similar to the profile of Rictor, Western blot analysis showed increased expression of Profilin-1 after birth, the 
lowest levels were detected at $\mathrm{P} 0$, and the highest levels were detected at P14 and maintained to P56 ( $\mathrm{p}<0.01$ when compared with P0 or P7), there were no significant differences among P14, P28 and P56, as shown in Fig. 1D.

\subsection{LET Dose-dependently Regulated SRC-1 and the F-actin/G-actin Ratio in vivo}

For steroid receptor coactivator-1 (SRC-1), the coactivator for the transcription of nuclear receptors, Western blot revealed that $40 \mu \mathrm{g} / \mathrm{kg}$ LET treatment did not induce any changes ( $\mathrm{p}>0.05$ when compared with control), and its expression was significantly downregulated by $80 \mu \mathrm{g} / \mathrm{kg}$ LET treatment without any further significant decrease at a dose of $160 \mu \mathrm{g} / \mathrm{kg}$ treatment (Fig. 2A).

The ratio of F-actin/G-actin has been regarded as indicator of the dynamics of actin polymerization [14]. The results showed that $40 \mu \mathrm{g} / \mathrm{kg}$ or $80 \mu \mathrm{g} / \mathrm{kg}$ LET treatment did not induce any changes in the F-actin/G-actin ratio, and it was significantly downregulated by $160 \mu \mathrm{g} / \mathrm{kg} \mathrm{LET}$ treatment, as indicated in Fig. 2B.

\subsection{LET Dose-dependently Regulated Actin Cytoskeleton Remodeling Proteins in vivo}

We first examined Rictor and its downstream effector phosphor-AKT ser 473 (p-AKT). The results showed that $40 \mu \mathrm{g} / \mathrm{kg}$ or $80 \mu \mathrm{g} / \mathrm{kg}$ LET treatment did not induce any changes in hippocampal Rictor or p-AKT. However, $160 \mu \mathrm{g} / \mathrm{kg}$ LET treatment downregulated Rictor and pAKT significantly ( $\mathrm{p}<0.01$ when compared to other groups). Additionally, the expression of total AKT was not changed by LET treatment. These results are shown in Fig. 2C-D.

For the expression of hippocampal Cofilin, Western blot analysis showed that its expression was slightly increased by LET treatment. However, levels of p-Cofilin were significantly changed after LET treatment. p-Cofilin/Cofilin ratio was dramatically downregulated by $80 \mu \mathrm{g} / \mathrm{kg}$ and 160 $\mu \mathrm{g} / \mathrm{kg}$ LET ( $<<0.01$ when compared with control and $40 \mu \mathrm{g} / \mathrm{kg}$ LET), and there was no significant difference between $80 \mu \mathrm{g} / \mathrm{kg}$ and $160 \mu \mathrm{g} / \mathrm{kg}$ LET treatment. These results are shown in Fig. 2E.

For the expression of hippocampal Profilin-1, Western blot analysis showed it was inhibited by LET treatment in a trend similar to that detected in Rictor. While $40 \mu \mathrm{g} / \mathrm{kg}$ and $80 \mu \mathrm{g} / \mathrm{kg}$ LET did not induce any changes, $160 \mu \mathrm{g} / \mathrm{kg}$ LET induced a significant decrease of Profilin- $1(\mathrm{p}<0.01$ for Western blot when compared with other groups) as shown in Fig. 2F.

\subsection{LET dose-dependently Downregulated SRC-1 and Actin Remodeling Proteins in vitro}

It has been shown that the embryonic mouse hippocampal cell line mHippoE-14 cells express nuclear estrogen receptor ER $\alpha$ and ER $\beta$ as well as membrane estrogen receptor GPR30 and many neuronal proteins [21]. We then tested the changes of SRC-1 and the above mentioned actin cytoskeleton proteins after different doses of LET treatment. As indicated in Fig. 3, compared with the DMSO control, levels of SRC-1, Rictor, p-AKT (ser473) and Profilin-1 were not changed under lower doses of LET $\left(10^{-8}\right.$ or $\left.10^{-10} \mathrm{M}\right)$, except for p-Cofilin, which was dramatically decreased by $10^{-8} \mathrm{M}$ LET. On the other hand, all of these proteins were significantly decreased by a high dose of LET $\left(10^{-6} \mathrm{M}\right)$, but total expression of AKT and total Cofilin was unchanged.

\section{5 siSRC-1 Downregulated Actin Remodeling Proteins in vitro}

We then tested the effect of SRC-1 inhibition on the expression of actin cytoskeleton remodeling proteins in mHippoE-14 cells. The results showed that empty lentivirus vector 
treatment (siCon) did not induce significant decrease of SRC-1 when compared with the untreated control cells (Con). For the individual proteins examined, siSRC-1 lentivirus treatment induced a significant decrease of SRC-1 (by approximately 70\%), Rictor (by approximately 67\%), pAKT/AKT (by approximately 62\%), p-Cofilin/Cofilin (by approximately 58\%) and Profilin-1 (by approximately 64\%). These results were shown in Fig. 4.

\subsection{The Effects of LET on SRC-1 and Actin Remodeling Proteins Were Reversed by E2 Administration in vitro}

To verify the effects of LET regulation on actin remodeling proteins were E2 dependent, we added E2 to the LET treated cells. The results showed while $10^{-6} \mathrm{~mol} / \mathrm{L}$ LET treated induced significant decrease of SRC-1 and actin remodeling proteins, levels of these proteins were significantly reversed by $10^{-6} \mathrm{~mol} / \mathrm{L}$ E2 treatment. These results were shown in Fig. 5.

\subsection{The Effects of E2 on Actin Remodeling Proteins Were Inhibited by siSRC-1 in vitro}

To explore the exact role of SRC-1 in the mediating E2 regulation on actin cytoskeleton remodeling, we first treated mHippoE-14 cells with E2 $10^{-6} \mathrm{~mol} / \mathrm{L}$ then transfected these cells with siSRC-1 lentiviruses. The results showed that levels of Rictor, p-AKT, Profilin-1 and p-Cofilin were significantly increased by E2 treatment, but this increase was significantly suppressed by siSRC-1 as shown in Fig. 6.

\section{Discussion}

E2 has been shown to play important roles in the regulation of morphological plasticity, such as spine density and synapse density [24-27], but the underlying mechanisms are still unclear. Since actin cytoskeleton polymerization has been regarded as the basis of dendritic spine plasticity, in this study, we first examined the postnatal profiles of AROM and selected actin cytoskeleton remodeling proteins (Rictor and its downstream effector p-AKT, Cofilin and p-Cofilin, Profilin-1) in the hippocampus of both sexes. We found that, generally, males and females showed similar profiles during postnatal development except female hippocampus tended to have higher expression of AROM. For these actin remodeling proteins, both males and females showed similar increased postnatal profiles. Therefore, in the adult hippocampus, higher levels of AROM and actin remodeling proteins were detected. Based on these findings we then investigated the effects of AROM inhibition on the expression of these actin cytoskeleton remodeling proteins as well as well as SRC-1, the steroid receptor coactivator which is highly expressed in the hippocampus, using AROM specific inhibitor LET. The in vivo and in vitro experiments demonstrated that when AROM was inhibited, levels of Rictor, p-AKT, Profilin-1 and p-Cofilin as well as SRC-1 were significantly decreased by LET treatment but reversed by E2 administration. Finally, we generated SRC-1 specific shRNA lentivirus and transfected hippocampal cells, the results showed levels of the above mentioned actin remodeling proteins were significantly decreased; and the E2 induced increase of actin cytoskeleton remodeling proteins was significantly suppressed by SRC-1 shRNA.

The exact levels of AROM and E2 concentrations in the hippocampus in males and females are still controversial. In the early 1980's, Roselli et al. reported trace amounts (negligible) of AROM enzymatic activity in rat brain regions including the hippocampus [28, 29]. Later studies demonstrated that, in developing rhesus monkeys and rodents, AROM activity was measurable mainly during late fetal and early postnatal development $[18,30,31]$. Balthazart et al. first 
examined AROM immunoreactivity in several birds and found the hippocampus was AROM negative [32], but Sanghera et al. reported moderate AROM immunoreactivity in the hippocampus of rats [33]. Later studies showed that, in the adult rodent hippocampus, the level of the AROM mRNA was about one-half of that detected in neonatal stages [17] or 1/300 of the ovary [3, 34]. However, recent studies have revealed that levels of hippocampus-derived E2 is significantly higher than the circulating levels, indicating hippocampal E2 is more important than circulating E2 in the regulation of the structure and function of hippocampus [35, 36]. We found higher levels of AROM protein were detected at early postnatal stages; it was dramatically decreased at from P7 to P28. Then it increased to a level comparable to early postnatal days in males except higher levels than early postnatal days in females in the adult (P56), indicating a V-type change of hippocampal E2 levels, especially in the females. Our results seemed different from Ivanova et al., who used semiquantitative reverse transcriptase PCR and revealed that AROM expression increased during the first two postnatal weeks and decreased to lower levels in adults [17] but agreement with Rune et al. [37] reporting that hippocampal E2 levels was low in males but high in females. Since both AROM activity and the concentration of hippocampal testosterone, the substrate of AROM, may contribute to the local E2 concentration, further detail work is urgently needed to elucidate the exact relationships between these factors; and the physiological implications regarding the postnatal profiles of hippocampal AROM in both sexes also need further exploration.

Cytoskeletal disorders have been related to many abnormalities such as neurodegenerative diseases [38]. The dynamics of the actin cytoskeleton have been shown to play a pivotal role in the proper development and functional connectivity of nerve tissue [39]. In synapses, F-actin plays a scaffolding role for the rapid reorganization of actin and it remodels the structure of the synapse during neuronal plasticity [40]. Many factors have been involved in the regulation of actin dynamics, in which Cofilin promotes the depolymerization while Profilin-1 promotes the elongation of actin [13]. Rictor is the regulatory protein of the mammalian target of rapamycin complex 2 (mTORC2) and it has been shown to be needed for proper dendritic arbor morphology [41]. A recent work by Huang et al. found that, when Rictor was conditionally deleted, actin polymerization was significantly reduced [14]. Because there is no available information about the expression of these actin remodeling proteins in the developing hippocampus, we then examined the postnatal profiles of selected key actin cytoskeleton regulatory proteins in the hippocampus. The results showed that during postnatal development, both male and female hippocampus shared similar profiles for the key actin regulatory proteins Rictor, Profilin-1 and Cofilin. The main difference seemed to be that, while the highest levels of Rictor and Profilin-1 were detected at P14, for p-Cofilin it was observed at P28. The parallel developmental profiles of these proteins in the hippocampus indicate their intrinsic potential in the regulation of actin remodeling and great contribution to the growth, maturation and plasticity of hippocampal neurons. However, their exact roles in the postnatal development of hippocampus also need further exploration.

The postnatal profiles of AROM and actin cytoskeleton remodeling proteins seemed different, considering the V-type profile of AROM but increased profiles of actin remodeling proteins. Additionally, in the adult hippocampus, female tended to have higher levels of AROM, but the profiles of actin remodeling proteins did not show obvious differences. The physiological significance of these phenomena was currently unknown. Since AROM functions not only to regulate spine density and synapse density [9] but also synaptic proteins [15] and the antiinflammatory and neuroprotective actions [42], the above discrepant profiles might indicate the 
functional complexity of hippocampal AROM beside its regulation on actin polymerization dynamics. Additionally, one may simply conclude that in the adult hippocampus, higher levels of AROM and actin remodeling proteins were detected; this still indicated a close relationship between AROM and actin remodeling proteins. However, the exact significances of these discrepancies are also needed to explore in the future.

To answer the question whether E2 can regulate actin dynamics and elucidate the related molecular mechanisms, we examined expressions of these key actin remodeling proteins after inhibition of local E2 synthesis by AROM inhibitor LET treatment. We found that when AROM activity was inhibited by LET, both Rictor and its downstream effector p-AKT, and Profilin-1 were inhibited by a higher dose of LET (160 $\mu \mathrm{g} / \mathrm{kg}$ B.W) and p-Cofilin were inhibited by a lower dose of LET ( $80 \mu \mathrm{g} / \mathrm{kg} \mathrm{B.W})$, indicating that local E2 plays an important role in the regulation of actin cytoskeleton remodeling. Importantly, we also found that the LET inhibition on actin cytoskeleton remodeling proteins could be significantly reversed by E2 administration, which provided solid evidences for the E2 regulation on actin cytoskeleton dynamics. So far rare studies reported E2 treatment on the expression of these actin cytoskeleton remodeling proteins, limited clues have shown that, in acute hippocampal slices, E2 treatment induced p-Cofilin and actin polymerization, which could be blocked by ER antagonists and activated by ER agonists [43]; while E2 synthesis inhibition induced dephosphorylation of Cofilin [37]. Our results were in good agreement with these previous studies and, to our knowledge, this is the first report showing direct evidence for estrogen regulation of Rictor and Profilin-1, although one study showed that, in mouse embryonic stem cells, expression of Profilin-1 was induced by E2 treatment [44].

The effects of $E 2$ have been shown to be mediated by its nuclear receptors (ER $\alpha$ and/or ER $\beta$ ) as well as membrane receptor GPER (also called GPR30) [2]. SRC-1 is the coactivator for nuclear receptors [45]. It has been reported that SRC-1 has multiple potentials in the hippocampus such as neural development and neural stem cell differentiation [4, 22, 46, 47]. SRC-1 knockout induced significant behavior deficiencies in a memory test [19], and it mediated LET regulation on hippocampal synaptic protein PSD-95 [15], indicating a role for this coactivator in the regulation of hippocampus function. To further explore the mechanism under local estrogen on hippocampal actin cytoskeleton plasticity, we first demonstrated that hippocampal SRC-1 was significantly regulated by LET treatment, which was in agreement with our previous reports $[15,22]$. Then, we generated SRC-1 RNA interference lentivirus and treated embryonic mouse hippocampal cell line mHippoE-14 cells. The results showed that when SRC-1 was inhibited by lentivirus transfection, levels of Rictor and p-AKT (ser473), the actin polymerization regulating proteins Profilin-1 and p-Cofilin were significantly downregulated. Moreover, we also found that the E2 induced increase of these proteins was significantly downregulated by SRC- 1 inhibition. These results clearly indicated both ER $\alpha$ and ER $\beta$ might be involved in the local E2 regulation on actin cytoskeleton plasticity and the important role of SRC-1 in the mediating of nuclear estrogen receptor action. Furthermore, our ongoing work showed that when hippocampal GPER was inactivated or activated by its specific antagonist G15 or agonist G1 in vivo or in vitro, levels of SRC-1 was also significantly downregulated (data not shown), indicating its "hub" role in the mediating of both nuclear and membrane ER action on actin cytoskeleton polymerization.

Ovary estrogens have been shown to regulate synapse density and spine density in the hippocampus of rodents $[24,25,48]$, thus playing a fundamental role in the regulation of 
cognition and memory. Local E2 has also been shown to regulate synaptic plasticity and cognition not only in rodents [49] but also in the human hippocampus [9]. However, the mechanisms are not clear. In this study, we demonstrated that local E2 plays an important role in the regulation of actin cytoskeleton dynamics, which might be mediated by SRC-1. These results clearly demonstrated that SRC-1 is directly involved in AROM/local E2 regulation on actin cytoskeleton remodeling and its role in the plasticity of dendritic spines, indicating its potential for the prevention and treatment of hippocampus-based disorders such as Alzheimer's disease (AD). Currently, results from existing studies using estrogen replacement to prevent and/or treat neurodegenerative dementias such as $\mathrm{AD}$ are equivocal regarding the benefits on cognition [50, 51], and levels of hippocampus-derived E2 is significantly higher than the circulating levels [35, 36]. Therefore, it will be very interesting and a great challenge to explore the exact roles of local E2 on neurodegenerative diseases.

\section{Acknowledgments}

This work was supported by the National Science Foundation of China (NSFC, No. 81571059).

\section{References cited}

[1] Y. Hara, E.M. Waters, B.S. McEwen, J.H. Morrison, Estrogen Effects on Cognitive and Synaptic Health Over the Lifecourse, Physiological reviews 95(3) (2015) 785-807.

[2] E. Simpson, R.J. Santen, Celebrating 75 years of oestradiol, Journal of molecular endocrinology 55(3) (2015) T1-20.

[3] Y. Hojo, T.A. Hattori, T. Enami, A. Furukawa, K. Suzuki, H.T. Ishii, H. Mukai, J.H. Morrison, W.G. Janssen, S. Kominami, N. Harada, T. Kimoto, S. Kawato, Adult male rat hippocampus synthesizes estradiol from pregnenolone by cytochromes P45017alpha and P450 aromatase localized in neurons, Proceedings of the National Academy of Sciences of the United States of America 101(3) (2004) 865-870.

[4] C. Bian, H. Zhu, Y. Zhao, W. Cai, J. Zhang, Intriguing roles of hippocampussynthesized 17beta-estradiol in the modulation of hippocampal synaptic plasticity, J Mol Neurosci 54(2) (2014) 271-281.

[5] O. Kretz, L. Fester, U. Wehrenberg, L. Zhou, S. Brauckmann, S. Zhao, J. Prange-Kiel, T. Naumann, H. Jarry, M. Frotscher, G.M. Rune, Hippocampal synapses depend on hippocampal estrogen synthesis, The Journal of neuroscience : the official journal of the Society for Neuroscience 24(26) (2004) 5913-5921.

[6] L. Fester, L. Zhou, A. Butow, C. Huber, R. von Lossow, J. Prange-Kiel, H. Jarry, G.M. Rune, Cholesterol-promoted synaptogenesis requires the conversion of cholesterol to estradiol in the hippocampus, Hippocampus 19(8) (2009) 692-705.

[7] S. Grassi, A. Tozzi, C. Costa, M. Tantucci, E. Colcelli, M. Scarduzio, P. Calabresi, V.E. Pettorossi, Neural 17beta-estradiol facilitates long-term potentiation in the hippocampal CA1 region, Neuroscience 192 (2011) 67-73.

[8] R. Vierk, J. Bayer, S. Freitag, M. Muhia, K. Kutsche, T. Wolbers, M. Kneussel, T. Sommer, G.M. Rune, Structure-function-behavior relationship in estrogen-induced synaptic plasticity, Hormones and behavior 74 (2015) 139-148.

[9] J. Bayer, G. Rune, H. Schultz, M.J. Tobia, I. Mebes, O. Katzler, T. Sommer, The effect of estrogen synthesis inhibition on hippocampal memory, Psychoneuroendocrinology 56 (2015) 213-225.

[10] N. Dominguez-Iturza, M. Calvo, M. Benoist, J.A. Esteban, M. Morales, Hippocampal Dendritic Spines Are Segregated Depending on Their Actin Polymerization, Neural Plast 2016 (2016) 2819107. 
[11] I. Hlushchenko, M. Koskinen, P. Hotulainen, Dendritic spine actin dynamics in neuronal maturation and synaptic plasticity, Cytoskeleton (Hoboken) (2016).

[12] J.T. Kevenaar, C.C. Hoogenraad, The axonal cytoskeleton: from organization to function, Frontiers in molecular neuroscience 8 (2015) 44.

[13] T.D. Pollard, G.G. Borisy, Cellular motility driven by assembly and disassembly of actin filaments, Cell 112(4) (2003) 453-465.

[14] W. Huang, P.J. Zhu, S. Zhang, H. Zhou, L. Stoica, M. Galiano, K. Krnjevic, G. Roman, M. Costa-Mattioli, mTORC2 controls actin polymerization required for consolidation of long-term memory, Nat Neurosci 16(4) (2013) 441-448.

[15] M. Liu, X. Huangfu, Y. Zhao, D. Zhang, J. Zhang, Steroid receptor coactivator-1 mediates letrozole induced downregulation of postsynaptic protein PSD-95 in the hippocampus of adult female rats, The Journal of steroid biochemistry and molecular biology 154 (2015) 168-175.

[16] R. Vierk, G. Glassmeier, L. Zhou, N. Brandt, L. Fester, D. Dudzinski, W. Wilkars, R.A. Bender, M. Lewerenz, S. Gloger, L. Graser, J. Schwarz, G.M. Rune, Aromatase inhibition abolishes LTP generation in female but not in male mice, The Journal of neuroscience : the official journal of the Society for Neuroscience 32(24) (2012) 8116-8126.

[17] T. Ivanova, C. Beyer, Ontogenetic expression and sex differences of aromatase and estrogen receptor-alpha/beta mRNA in the mouse hippocampus, Cell and tissue research 300(2) (2000) 231-237.

[18] N.J. MacLusky, M.J. Walters, A.S. Clark, C.D. Toran-Allerand, Aromatase in the cerebral cortex, hippocampus, and mid-brain: ontogeny and developmental implications, Mol Cell Neurosci 5(6) (1994) 691-698.

[19] E. Nishihara, H. Yoshida-Komiya, C.S. Chan, L. Liao, R.L. Davis, B.W. O'Malley, J. $\mathrm{Xu}, \mathrm{SRC}-1$ null mice exhibit moderate motor dysfunction and delayed development of cerebellar Purkinje cells, The Journal of neuroscience : the official journal of the Society for Neuroscience 23(1) (2003) 213-222.

[20] L. Naldini, U. Blomer, P. Gallay, D. Ory, R. Mulligan, F.H. Gage, I.M. Verma, D. Trono, In vivo gene delivery and stable transduction of nondividing cells by a lentiviral vector, Science 272(5259) (1996) 263-267.

[21] S. Gingerich, G.L. Kim, J.A. Chalmers, M.M. Koletar, X. Wang, Y. Wang, D.D. Belsham, Estrogen receptor alpha and G-protein coupled receptor 30 mediate the neuroprotective effects of 17 beta-estradiol in novel murine hippocampal cell models, Neuroscience 170(1) (2010) 54-66.

[22] C. Bian, Y. Zhao, Q. Guo, Y. Xiong, W. Cai, J. Zhang, Aromatase inhibitor letrozole downregulates steroid receptor coactivator-1 in specific brain regions that primarily related to memory, neuroendocrine and integration, The Journal of steroid biochemistry and molecular biology 141 (2014) 37-43.

[23] L. Qiu, Y. Zhao, Q. Guo, Y. Zhang, L. He, W. Li, J. Zhang, Dose-dependent regulation of steroid receptor coactivator-1 and steroid receptors by testosterone propionate in the hippocampus of adult male mice, J Steroid Biochem Mol Biol 156 (2016) 23-31.

[24] C.S. Woolley, B.S. McEwen, Estradiol mediates fluctuation in hippocampal synapse density during the estrous cycle in the adult rat, The Journal of neuroscience : the official journal of the Society for Neuroscience 12(7) (1992) 2549-2554.

[25] C.S. Woolley, B.S. McEwen, Estradiol regulates hippocampal dendritic spine density via an N-methyl-D-aspartate receptor-dependent mechanism, The Journal of neuroscience : the official journal of the Society for Neuroscience 14(12) (1994) 7680-7687.

[26] C.S. Woolley, H.J. Wenzel, P.A. Schwartzkroin, Estradiol increases the frequency of multiple synapse boutons in the hippocampal CA1 region of the adult female rat, The Journal of comparative neurology 373(1) (1996) 108-117.

[27] L.F. Jacome, K. Barateli, D. Buitrago, F. Lema, M. Frankfurt, V.N. Luine, Gonadal hormones rapidly enhance spatial memory and increase hippocampal spine density in male rats, Endocrinology (2016) en20151959. 
[28] J. Prange-Kiel, D.A. Dudzinski, F. Prols, M. Glatzel, J. Matschke, G.M. Rune, Aromatase Expression in the Hippocampus of AD Patients and 5xFAD Mice, Neural Plast 2016 (2016) 9802086.

[29] C.E. Roselli, W.E. Ellinwood, J.A. Resko, Regulation of brain aromatase activity in rats, Endocrinology 114(1) (1984) 192-200.

[30] C.E. Roselli, L.E. Horton, J.A. Resko, Distribution and regulation of aromatase activity in the rat hypothalamus and limbic system, Endocrinology 117(6) (1985) 2471-2477.

[31] Y. Takahashi, H. Yamanaka, S. Honma, [The regulation system of brain aromatase activity; distribution and changes with age of the aromatase activity of the rat brain], Nihon Naibunpi Gakkai Zasshi 63(7) (1987) 862-869.

[32] J. Balthazart, A. Foidart, C. Surlemont, A. Vockel, N. Harada, Distribution of aromatase in the brain of the Japanese quail, ring dove, and zebra finch: an immunocytochemical study, The Journal of comparative neurology 301(2) (1990) 276-288.

[33] M.K. Sanghera, E.R. Simpson, M.J. McPhaul, G. Kozlowski, A.J. Conley, E.D. Lephart, Immunocytochemical distribution of aromatase cytochrome P450 in the rat brain using peptide-generated polyclonal antibodies, Endocrinology 129(6) (1991) 2834-2844.

[34] G. Murakami, N. Tanabe, H.T. Ishii, M. Ogiue-Ikeda, T. Tsurugizawa, H. Mukai, Y. Hojo, N. Takata, A. Furukawa, T. Kimoto, S. Kawato, Role of cytochrome p450 in synaptocrinology: endogenous estrogen synthesis in the brain hippocampus, Drug metabolism reviews 38(3) (2006) 353-369.

[35] Y. Hojo, S. Higo, H. Ishii, Y. Ooishi, H. Mukai, G. Murakami, T. Kominami, T. Kimoto, S. Honma, D. Poirier, S. Kawato, Comparison between hippocampus-synthesized and circulation-derived sex steroids in the hippocampus, Endocrinology 150(11) (2009) 51065112.

[36] Y. Ooishi, S. Kawato, Y. Hojo, Y. Hatanaka, S. Higo, G. Murakami, Y. Komatsuzaki, M. Ogiue-Ikeda, T. Kimoto, H. Mukai, Modulation of synaptic plasticity in the hippocampus by hippocampus-derived estrogen and androgen, The Journal of steroid biochemistry and molecular biology 131(1-2) (2012) 37-51.

[37] R. Vierk, N. Brandt, G.M. Rune, Hippocampal estradiol synthesis and its significance for hippocampal synaptic stability in male and female animals, Neuroscience 274 (2014) 2432.

[38] J.R. Bamburg, B.W. Bernstein, Actin dynamics and cofilin-actin rods in Alzheimer disease, Cytoskeleton (Hoboken) (2016).

[39] S. Menon, S.L. Gupton, Building Blocks of Functioning Brain: Cytoskeletal Dynamics in Neuronal Development, International review of cell and molecular biology 322 (2016) 183-245.

[40] L.A. Cingolani, Y. Goda, Actin in action: the interplay between the actin cytoskeleton and synaptic efficacy, Nature reviews. Neuroscience 9(5) (2008) 344-356.

[41] M. Urbanska, A. Gozdz, L.J. Swiech, J. Jaworski, Mammalian target of rapamycin complex 1 (mTORC1) and 2 (mTORC2) control the dendritic arbor morphology of hippocampal neurons, The Journal of biological chemistry 287(36) (2012) 30240-30256.

[42] Q.G. Zhang, R. Wang, H. Tang, Y. Dong, A. Chan, G.R. Sareddy, R.K. Vadlamudi, D.W. Brann, Brain-derived estrogen exerts anti-inflammatory and neuroprotective actions in the rat hippocampus, Molecular and cellular endocrinology 389(1-2) (2014) 84-91.

[43] V. Briz, M. Baudry, Estrogen Regulates Protein Synthesis and Actin Polymerization in Hippocampal Neurons through Different Molecular Mechanisms, Frontiers in endocrinology 5 (2014) 22.

[44] S.P. Yun, J.M. Ryu, M.O. Kim, J.H. Park, H.J. Han, Rapid actions of plasma membrane estrogen receptors regulate motility of mouse embryonic stem cells through a profilin-1/cofilin-1-directed kinase signaling pathway, Molecular endocrinology (Baltimore, Md.) 26(8) (2012) 1291-1303.

[45] J. Xu, Q. Li, Review of the in vivo functions of the p160 steroid receptor coactivator family, Molecular endocrinology (Baltimore, Md.) 17(9) (2003) 1681-1692. 
[46] E. Nishihara, T. Moriya, K. Shinohara, Expression of steroid receptor coactivator-1 is elevated during neuronal differentiation of murine neural stem cells, Brain research 1135(1) (2007) 22-30.

[47] D. Zhang, Q. Guo, C. Bian, J. Zhang, S. Lin, B. Su, Alterations of steroid receptor coactivator-1 (SRC-1) immunoreactivities in specific brain regions of young and middle-aged female Sprague-Dawley rats, Brain research 1382 (2011) 88-97.

[48] B.S. McEwen, C.S. Woolley, Estradiol and progesterone regulate neuronal structure and synaptic connectivity in adult as well as developing brain, Experimental gerontology 29(3-4) (1994) 431-436.

[49] J.J. Tuscher, J.S. Szinte, J.R. Starrett, A.A. Krentzel, A.M. Fortress, L. RemageHealey, K.M. Frick, Inhibition of local estrogen synthesis in the hippocampus impairs hippocampal memory consolidation in ovariectomized female mice, Hormones and behavior 83 (2016) 60-67.

[50] C.E. Gleason, N.M. Dowling, W. Wharton, J.E. Manson, V.M. Miller, C.S. Atwood, E.A. Brinton, M.I. Cedars, R.A. Lobo, G.R. Merriam, G. Neal-Perry, N.F. Santoro, H.S. Taylor, D.M. Black, M.J. Budoff, H.N. Hodis, F. Naftolin, S.M. Harman, S. Asthana, Effects of Hormone Therapy on Cognition and Mood in Recently Postmenopausal Women: Findings from the Randomized, Controlled KEEPS-Cognitive and Affective Study, PLoS Med 12(6) (2015) e1001833; discussion e1001833.

[51] B. Fischer, C. Gleason, S. Asthana, Effects of hormone therapy on cognition and mood, Fertility and sterility 101(4) (2014) 898-904. 
Figure legend

Figure 1. Postnatal developmental profiles of aromatase and actin cytoskeleton remodeling proteins in the hippocampus of mice. A: Profiles of aromatase. Higher levels of aromatase were detected before P7, and then it decreased significantly from P14 to P28. At P56, it increased to the $\mathrm{P} 0$ or P7 levels in males but increased to a higher level than P0 or P7 in the females. **: $\mathrm{p}<0.01$ when compared with P0, P7 and P56 in the males or P0 and P7 in the females. B: Profiles of Rictor. In both sexes, the lowest levels were detected at P0, and then it increased gradually and reached the highest levels at P14. There were no differences detected among P14, P28 and P56. **: $\mathrm{p}<0.01$ when compared to P14. C: Profiles of p-Cofilin and Cofilin. The levels of Cofilin were almost unchanged but $\mathrm{p}$-Cofilin changed significantly. The ratio of $\mathrm{p}$-Cofilin/Cofilin was the lowest at P0, and then it increased gradually and reached the highest levels at P28, then decreased slightly at P56. *: $\mathrm{p}<0.05, * *$ : $\mathrm{p}<0.01$, compared with P28. D: Profiles of Profilin-1. In both sexes, the lowest levels were detected at P0, and then it increased gradually and reached the highest levels at P14. There were no differences detected among P14, P28 and P56. **: p<0.01 when compared with p14, P28 or P56.

Figure 2. Dose-dependent regulation of letrozole (LET) on SRC-1, F-actin/G-actin and actin cytoskeleton remodeling proteins in the hippocampus of female mice. A: SRC-1 was significantly decreased by $80 \mu \mathrm{g} / \mathrm{kg}$ and $160 \mu \mathrm{g} / \mathrm{kg}$ LET. There was no significant difference between DMSO and a low dose of LET (40 $\mu \mathrm{g} / \mathrm{kg}$ B.W), or between $80 \mu \mathrm{g} / \mathrm{kg}$ and $160 \mu \mathrm{g} / \mathrm{kg}$ LET. ${ }^{* *}$ : p $<0.01$ when compared with DMSO. B: The F-actin/G-actin ratio was significantly decreased by 160 $\mu \mathrm{g} / \mathrm{kg}$ LET treatment. There were no significant differences among the other three groups. $* *$ : p $<0.01$ when compared with DMSO. C: levels of Rictor were significantly decreased by 160 $\mu \mathrm{g} / \mathrm{kg}$ LET treatment. There were no significant differences among the other three groups. ${ }^{* *}$ : $\mathrm{p}<0.01$ when compared with DMSO. D: Changes of total AKT and p-AKT. While total AKT showed no changed by any dose of LET, p-AKT was significantly decreased by a high dose of LET $(160 \mu \mathrm{g} / \mathrm{kg} \mathrm{B.W})$. There were no significant differences among the other three groups. **: $\mathrm{p}<0.01$ when compared to DMSO. E: Changes of total Cofilin and p-Cofilin. Total Cofilin was slightly increased by $160 \mu \mathrm{g} / \mathrm{kg}$ LET treatment and p-Cofilin was significantly decreased by 80 $\mu \mathrm{g} / \mathrm{kg}$ and $160 \mu \mathrm{g} / \mathrm{kg}$ LET, thus the $\mathrm{p}$-Cofilin/Cofilin ratio was significantly decreased by $80 \mu \mathrm{g} / \mathrm{kg}$ and $160 \mu \mathrm{g} / \mathrm{kg}$ LET. **: p $<0.01$ when compared with DMSO. F: Changes of Profilin-1 after LET treatment. Lower dose of LET ( $40 \mu \mathrm{g} / \mathrm{kg}$ and $80 \mu \mathrm{g} / \mathrm{kg} \mathrm{B.W}$ ) did not induce significant decrease of Profilin-1, and a higher dose of LET (160 $\mu \mathrm{g} / \mathrm{kg} \mathrm{B.W})$ induced a significant decrease of Profilin-1. **: $\mathrm{p}<0.01$ when compared with DMSO.

Figure 3. Dose-dependent regulation of LET on SRC-1 and actin cytoskeleton remodeling proteins in the embryonic mouse hippocampal cell line mHippoE-14 cells. A: SRC-1 was significantly decreased by $10^{-6}$ M LET. B: Rictor was significantly decreased by $10^{-6}$ M LET. C: p-AKT was significantly decreased by $10^{-6} \mathrm{M}$ LET while AKT unchanged, thus the p-AKT/AKT ratio was significantly decreased by $10^{-6} \mathrm{M}$ LET. D: p-Cofilin was significantly decreased by $10^{-6}$ and $10^{-8}$ M LET but Cofilin was unchanged, leading to a decreased $\mathrm{p}$-Cofilin/Cofilin ratio with $10^{-6}$ and $10^{-}$ ${ }^{8}$ M LET treatments. E: Profilin-1 was significantly decreased by $10^{-6} \mathrm{M}$ LET. There were no significant differences detected among DMSO, $10^{-8}$ and $10^{-10}$ M LET (A, B, C and E) or between 
DMSO and $10^{-10}$ M LET (D). **: $\mathrm{p}<0.01$ when compared with DMSO, $10^{-8}$ and $10^{-10}$ M LET (A, $\mathrm{B}, \mathrm{C}$ and E) or with DMSO and $10^{-10} \mathrm{M}$ LET (D).

Figure 4. Effects of SRC-1 knockdown on the expression of actin cytoskeleton remodeling proteins in mHippoE-14 cells. A: SRC-1 was successfully inhibited by specific RNA interference. B: Rictor was significantly decreased by SRC-1 knockdown. C: p-AKT was significantly decreased by SRC-1 knockdown while AKT was unchanged, thus the p-AKT/AKT ratio was significantly decreased. D: p-Cofilin was significantly decreased by SRC-1 knockdown but Cofilin was unchanged, leading to a decreased p-Cofilin/Cofilin ratio. E: Profilin-1 was significantly decreased by SRC-1 knockdown. Con: normal control. siCon: lentivirus vector control. siSRC-1: shSRC-1-containing lentivirus. ${ }^{* *}: \mathrm{p}<0.01$ when compared with the other groups.

Figure 5. 17ß-estradiol (E2) treatment significantly reversed the effects of letrozole (LET) induced downregulation of SRC-1 and actin cytoskeleton remodeling proteins in vitro. The concentration of E2 and LET was $10^{-6} \mathrm{~mol} / \mathrm{L}$, respectively. A: Levels of SRC-1 were reversed by E2. B-C: Rictor and p-AKT were reversed by E2. D-E: p-Cofilin and Profilin-1 were reversed by E2. **: $\mathrm{p}<0.01$ when compared with the other two groups.

Figure 6. 17- $\beta$ estradiol (E2) induced increase of actin cytoskeleton proteins were suppressed by SRC-1 interference (siSRC-1). The concentration of E2 was $10^{-6} \mathrm{~mol} / \mathrm{L}$. A: E2 induced increase of SRC-1 was inhibited by siSRC-1. B-C: E2 induced increase of Rictor and p-AKT was inhibited by siSRC-1. D-E: E2 induced increase of p-Cofilin and Profilin-1 was inhibited by siSRC-1. siCon: empty lentivirus vector. siSRC-1: shSRC-1-containing lentivirus. *: $\mathrm{p}<0.05$ when compared with siCon. **: $p<0.01$ when compared with the other groups. 


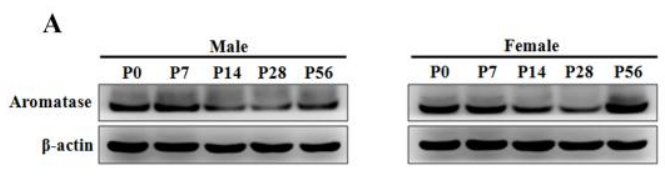

B
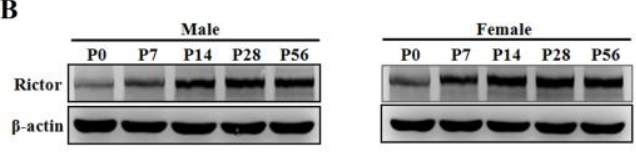

更

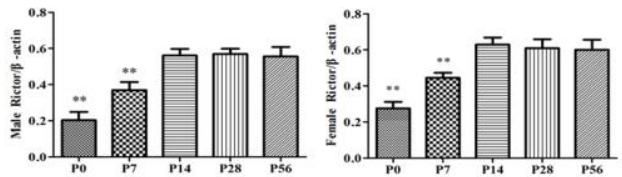

C

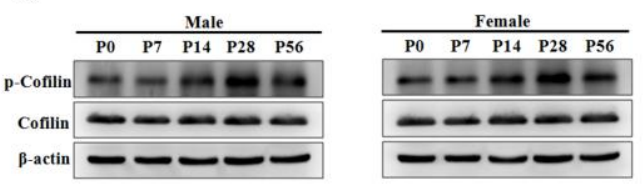

D

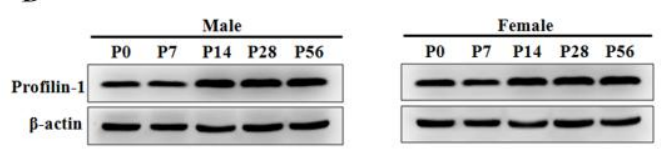

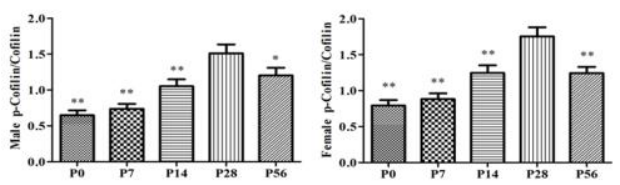

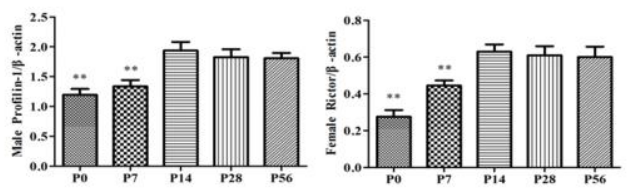



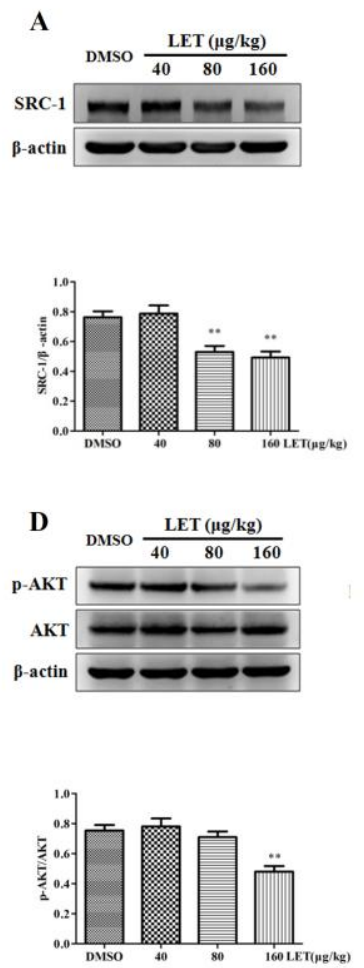

B
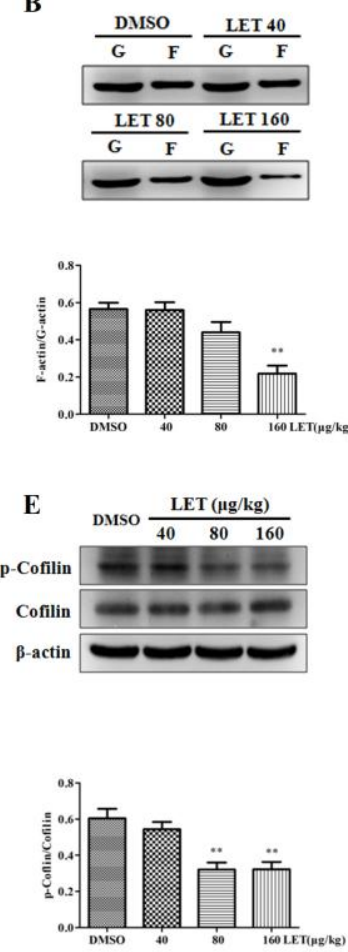

C
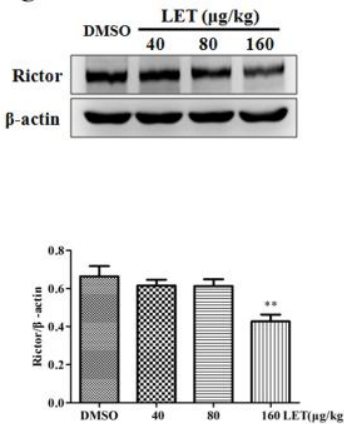

F DMSO $\frac{\text { LET }(\mu \mathrm{g} / \mathrm{kg})}{40 \quad 80 \quad 160}$
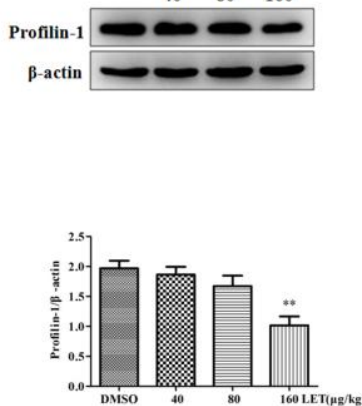

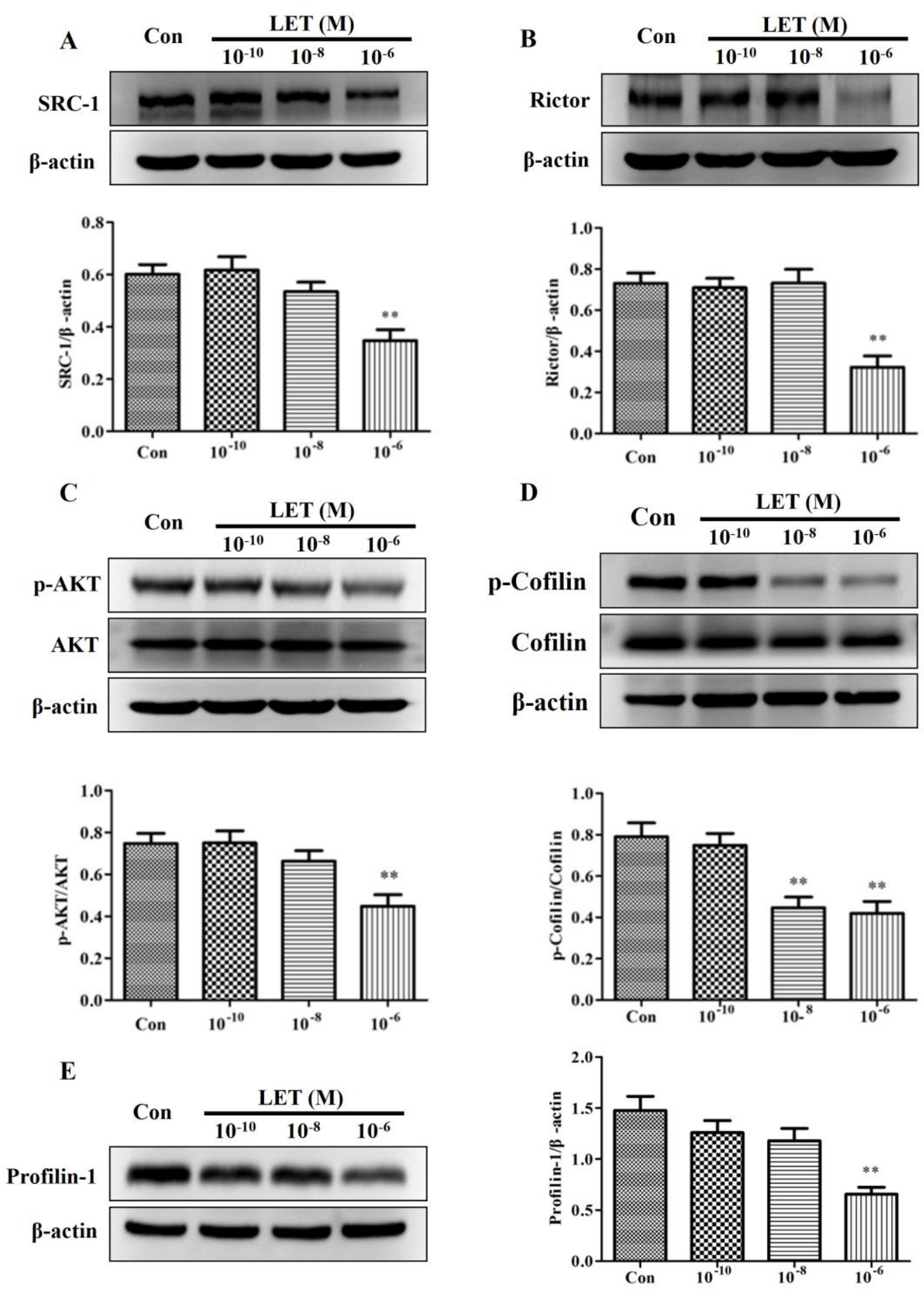
A
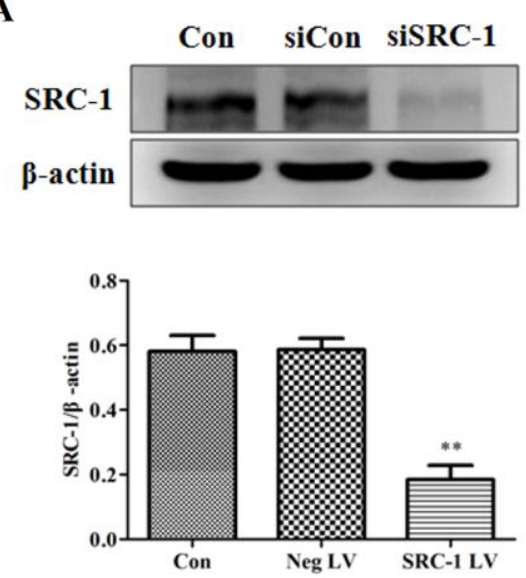

C
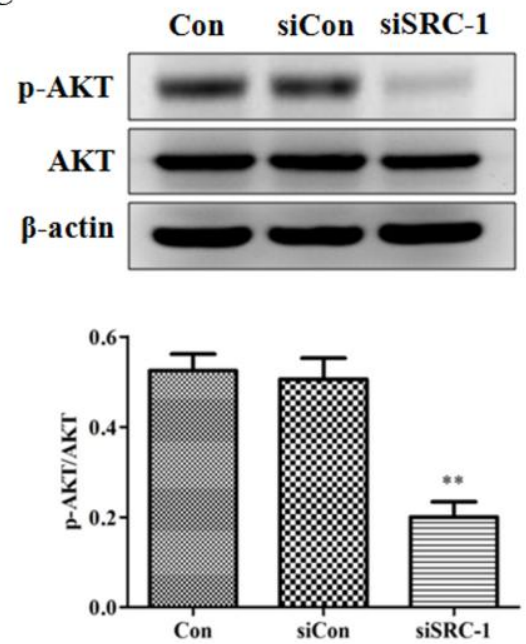

E

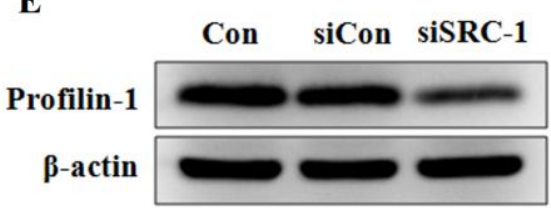

B
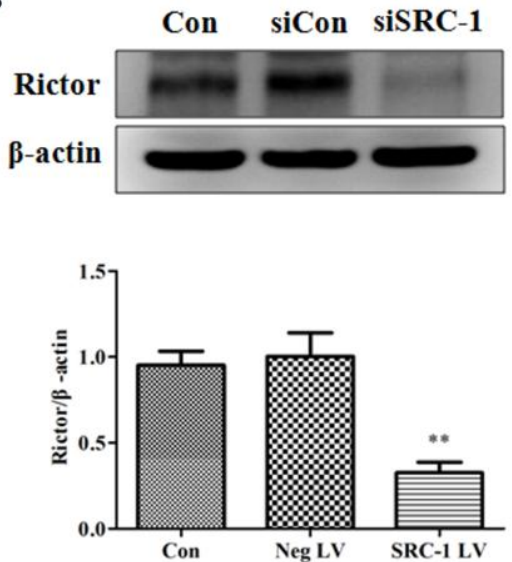

D
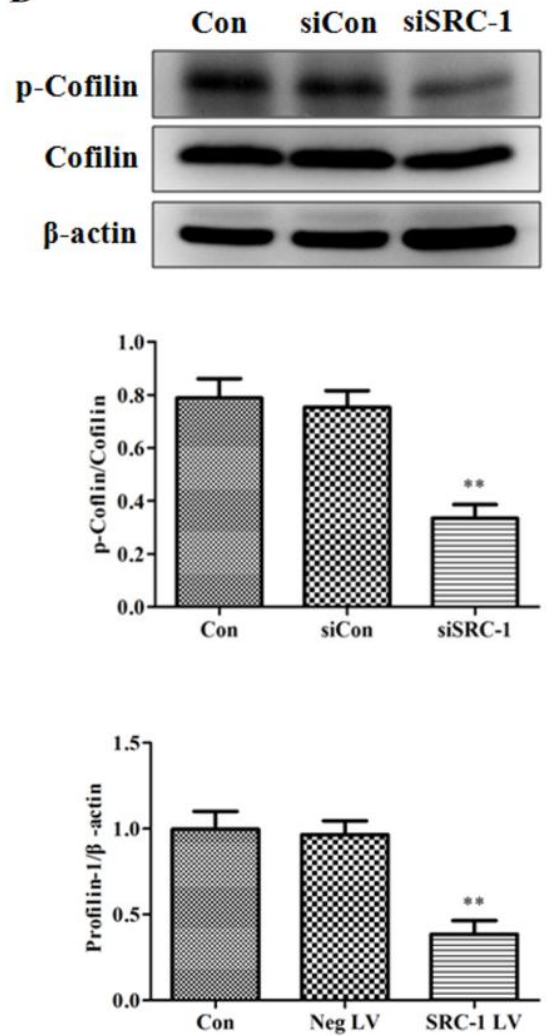
A
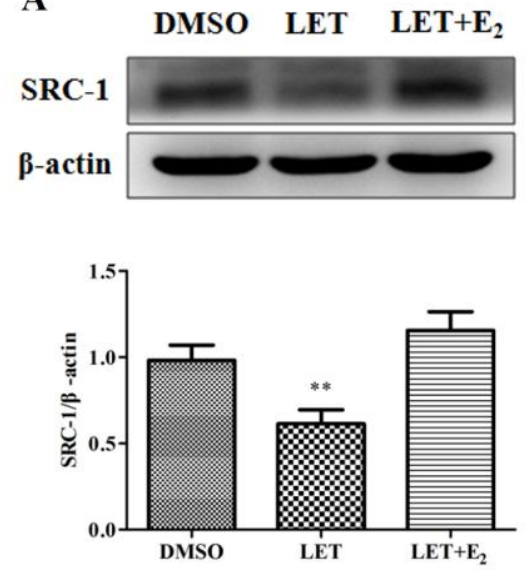

C

DMSO LET LET $+\mathrm{E}_{2}$
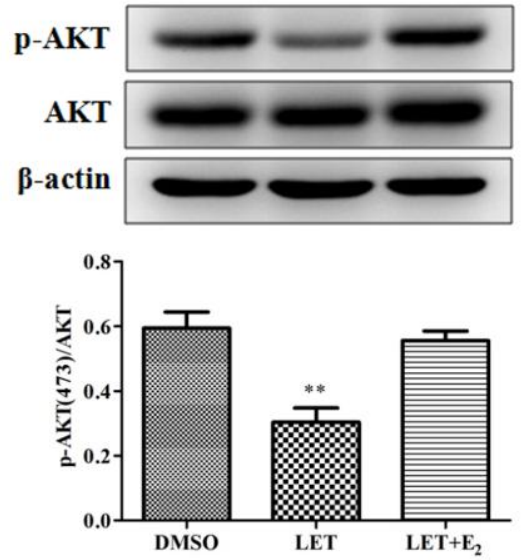

E DMSO LET LET $+\mathrm{E}_{2}$

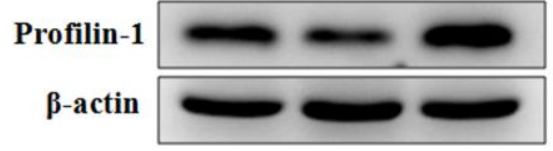

B

DMSO LET LET $+\mathbf{E}_{2}$
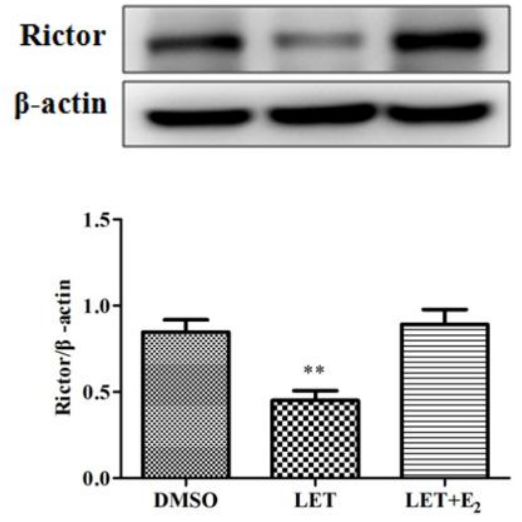

D
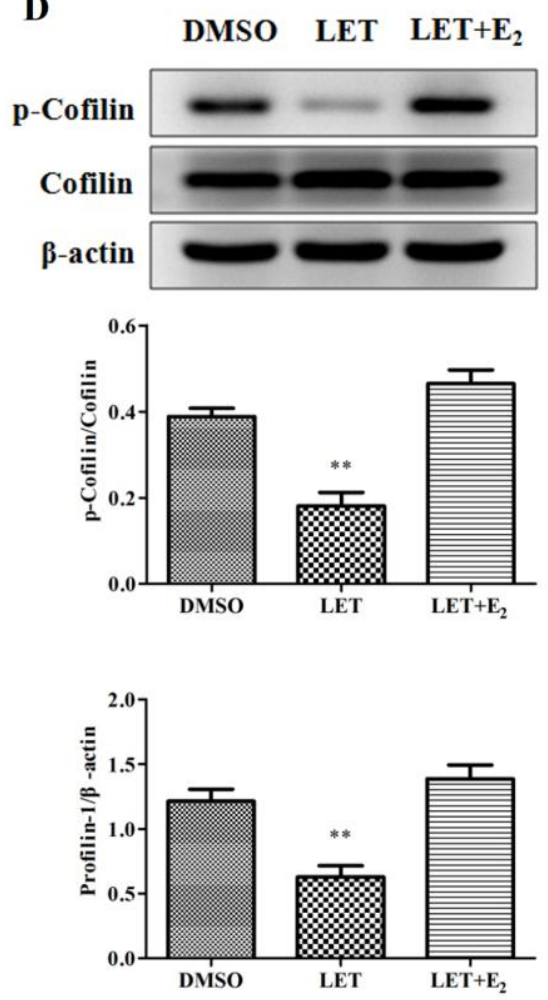

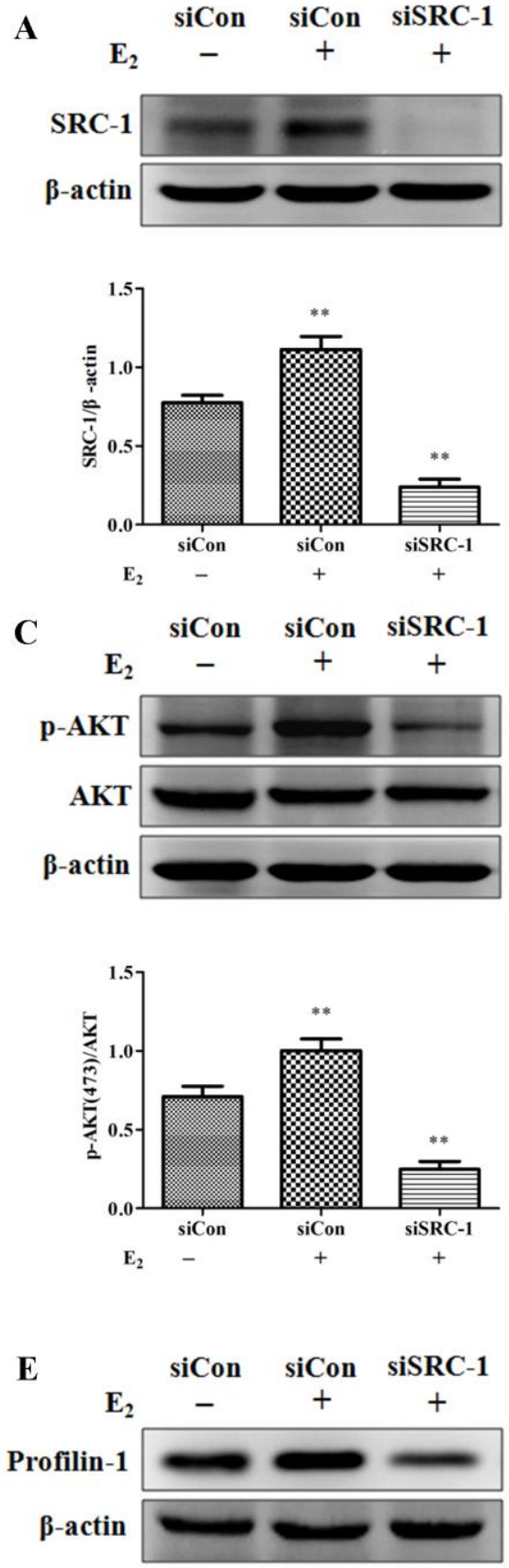
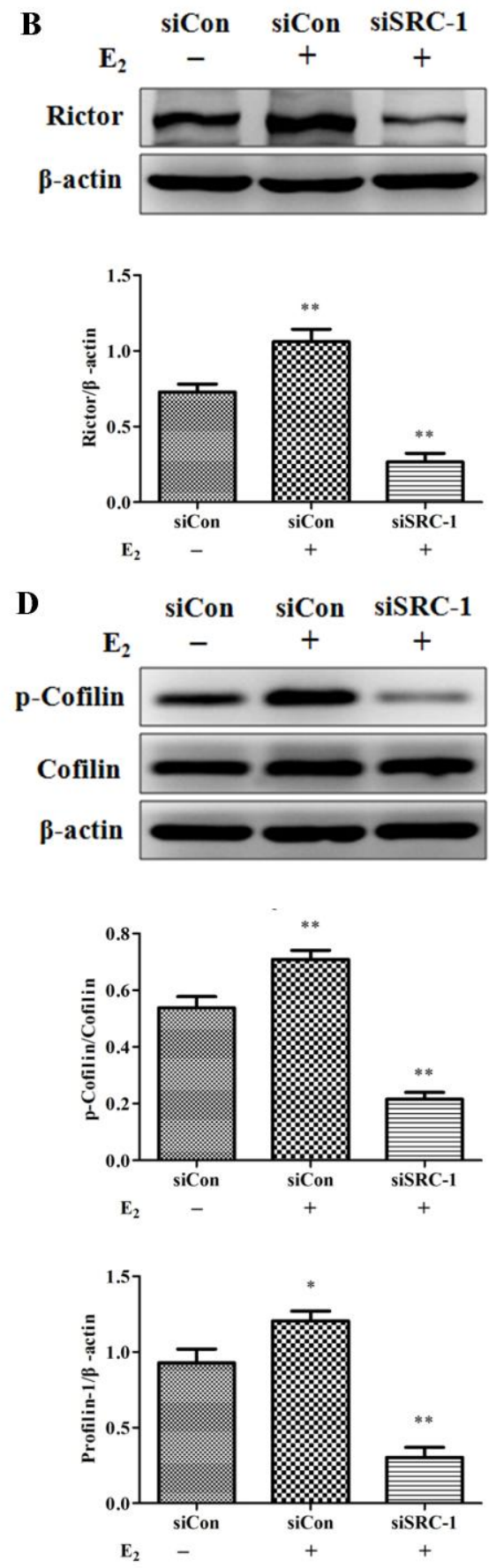